\title{
Closed-loop Wavefront Correction for High Contrast Imaging: The "peak-a-boo" Algorithm
}

\author{
Amir Give'on ${ }^{1} \dagger$, N. Jeremy Kasdin ${ }^{1}$, and Robert J. Vanderbei ${ }^{2}$ \\ ${ }^{1}$ Department of Mechanical and Aerospace Engineering, \\ Princeton University, Princeton, NJ 08544 \\ ${ }^{2}$ Department of Operational Research and Financial Engineering, \\ Princeton University, Princeton, NJ 08544
}

\begin{abstract}
High contrast imaging from space must overcome photon noise of the diffracted star light and scattered light from optical components defects. The very high contrast required (up to $10^{-10}$ for terrestrial planets) puts severe requirements on the wavefront control system, as the achievable contrast is limited by the quality of the wavefront. In this paper we present a closed loop correction method for the shaped pupil coronagraph to minimize the energy in a pre-defined region in the image where terrestrial planets would be found. The reconstruction part uses three intensity measurements in the image plane with a pinhole added to the shaped pupil for diversity. This method has been shown in simulations to be effective in the presence of photon noise, deformable mirror modeling errors and overcoming defective actuators in the deformable mirror.
\end{abstract}

Keywords. instrumentation: adaptive optics.

\section{Introduction}

Recently, the problem of high-contrast imaging (typically using a coronagraph) has become of increasing interest. Here, we refer to the problem of imaging a very dim point source very near a much brighter point or extended source (star). There are numerous missions planned for both ground and space to search for planets and other objects close to nearby stars [Beichman (1999)]. Our group at Princeton University has been pioneering the Shaped Pupil Coronagraph (SPC) [Kasdin (2003)]. This imaging system uses a binary apodization mask (shaped pupil) to shape the point spread function (PSF) such that a predefined region in the image plane will have the high contrast needed. The very high contrast required (up to $10^{-10}$ for terrestrial planets) puts severe requirements on the wavefront control system, as the achievable contrast is limited by the quality of the wavefront.

The performance of conventional AO systems typically has been limited by the accuracy of the wavefront phase estimation (known as phase reconstruction) and the ability of the deformable mirror (DM) to achieve the arbitrary shapes required [Tyson (2000)]. However, as improvements have been made in both these areas, the ability to achieve the deep nulls required for high contrast imaging has still been stalled [Poyneer (2004)]. We showed in [Give'on (2005)] that this is due to what we refer to as frequency folding. We demonstrated that even with a perfect estimation of the wavefront and a DM limited

$\dagger$ Michelson graduate fellow, Michelson Science Center, California Institute of Technology, 770 S Wilson Avenue, MS 100-22, Pasadena, CA 91125 
only by the highest spatial frequency it can achieve, conventional phase conjugation is limited by intermodulation products of its high frequency content.

In this paper we present a closed loop correction method for the shaped pupil coronagraph to minimize the energy in a pre-defined region in the image where terrestrial planets would be found. The correction part of the method is based on [Borde' (2005)]. The innovative reconstruction part uses three intensity measurements in the image plane with a pinhole added to the shaped pupil for diversity.

\section{Wavefront Reconstruction - The "peak-a-boo" Algorithm}

The electric field at the image plane, in the presence of aberrations in the system is a complex valued function. The measured quantity by the CCD is proportional to the intensity of the field where the complex field information is lost. The wavefront reconstruction problem is to recover of the real and imaginary parts of the complex field at the image plane.

Given a shaped pupil described by $A(x, y)$, The electric field in the image plane for the aberrated system operating in monochromatic light with wavelength $\lambda$ and a focal length $f$, is given by:

$$
\begin{aligned}
E(\xi, \eta) & =\frac{1}{\lambda f} \int_{-\infty}^{\infty} \int_{-\infty}^{\infty} A(x, y) e^{\alpha(x, y)+\frac{2 \pi}{\lambda} \beta(x, y)} e^{-i \frac{2 \pi}{\lambda f}(x \xi+y \eta)} d x d y \\
& =\frac{1}{\lambda f} \int_{-\infty}^{\infty} \int_{-\infty}^{\infty} A(x, y)[1+\Phi(x, y)] e^{-i \frac{2 \pi}{\lambda f}(x \xi+y \eta)} d x d y
\end{aligned}
$$

where we define

$$
\Phi(x, y)=e^{\alpha(x, y)+\frac{2 \pi}{\lambda} \beta(x, y)}-1
$$

which can be written as:

$$
\begin{aligned}
E(\xi, \eta) & =E_{0}(\xi, \eta)+E_{\Phi}(\xi, \eta) \\
& =E_{0}(\xi, \eta)+E_{\Phi}^{R}(\xi, \eta)+i E_{\Phi}^{I}(\xi, \eta)
\end{aligned}
$$

where $E_{\Phi}^{R}(\xi, \eta)$ and $E_{\Phi}^{I}(\xi, \eta)$ are the real and imaginary parts of $E_{\Phi}(\xi, \eta)$, respectively, and

$$
\begin{aligned}
& E_{0}(\xi, \eta)=\frac{1}{\lambda f} \int_{-\infty}^{\infty} \int_{-\infty}^{\infty} A(x, y) e^{-i \frac{2 \pi}{\lambda f}(x \xi+y \eta)} d x d y \\
& E_{\Phi}(\xi, \eta)=\frac{1}{\lambda f} \int_{-\infty}^{\infty} \int_{-\infty}^{\infty} A(x, y) \Phi(x, y) e^{-i \frac{2 \pi}{\lambda f}(x \xi+y \eta)} d x d y
\end{aligned}
$$

Since $E_{0}(\xi, \eta)$ is theoretically known (since we know $A(x, y)$ ), the reconstruction problem is to recover $E_{\Phi}^{R}(\xi, \eta)$ and $E_{\Phi}^{I}(\xi, \eta)$.

The diversity for image plane measurements suggested here is a pinhole in the center of the shaped pupil and using the actuators on the DM that are aligned with the pinhole location to change the phase across it. Let the complex valued function $h_{k}(x, y)(k=$ $1,2,3)$ denote the electric field due to the pinhole and the phase difference across it induced by the DM, then the corresponding electric field at the image plane in each case, is given by:

$$
\begin{aligned}
E_{k}(\xi, \eta) & =\frac{1}{\lambda f} \int_{-\infty}^{\infty} \int_{-\infty}^{\infty}\left\{A(x, y)[1+\Phi(x, y)]+h_{k}(x, y)\right\} e^{-i \frac{2 \pi}{\lambda f}(x \xi+y \eta)} d x d y \\
& =E_{0}(\xi, \eta)+E_{\Phi}^{R}(\xi, \eta)+i E_{\Phi}^{I}(\xi, \eta)+\Delta_{k}(\xi, \eta)
\end{aligned}
$$


where,

$$
\Delta_{k}(\xi, \eta)=\frac{1}{\lambda f} \int_{-\infty}^{\infty} \int_{-\infty}^{\infty} h_{k}(x, y) e^{-i \frac{2 \pi}{\lambda f}(x \xi+y \eta)} d x d y
$$

and the three PSF's are given by:

then, if we let

$$
I_{k}=E_{k}^{*} E_{k}
$$

$$
\begin{aligned}
G_{12} & =I_{2}-I_{1}-\Delta_{2}^{*} \Delta_{2}+\Delta_{1}^{*} \Delta_{1}-2 \Re\left\{E_{0} \Delta_{2}\right\}+2 \Re\left\{E_{0} \Delta_{1}\right\} \\
G_{13} & =I_{3}-I_{1}-\Delta_{3}^{*} \Delta_{3}+\Delta_{1}^{*} \Delta_{1}-2 \Re\left\{E_{0} \Delta_{3}\right\}+2 \Re\left\{E_{0} \Delta_{1}\right\} \\
A_{1} & =2\left(\Re\left\{\Delta_{2}\right\}-\Re\left\{\Delta_{1}\right\}\right) \\
A_{2} & =2\left(\Im\left\{\Delta_{2}\right\}-\Im\left\{\Delta_{1}\right\}\right) \\
A_{3} & =2\left(\Re\left\{\Delta_{3}\right\}-\Re\left\{\Delta_{1}\right\}\right) \\
A_{4} & =2\left(\Im\left\{\Delta_{3}\right\}-\Im\left\{\Delta_{1}\right\}\right)
\end{aligned}
$$

then the real and imaginary parts of $E_{\Phi}(\xi, \eta)$ are given by:

$$
E_{\Phi}^{R}=\frac{A_{4} G_{12}-A_{2} G_{13}}{A_{1} A_{4}-A_{2} A_{3}}, \quad E_{\Phi}^{I}=\frac{A_{1} G_{13}-A_{3} G_{12}}{A_{1} A_{4}-A_{2} A_{3}}
$$

\section{Wavefront Correction}

Let the DM be modeled with $N \times N$ actuators on a square grid and its surface, $\phi(x, y)$, following the super position principle,

$$
\phi(x, y)=\sum_{k=0}^{N-1} \sum_{l=0}^{N-1} a_{k, l} f_{k, l}(x, y)
$$

where $a_{k, l}$ is the height of the actuator on the $k^{t h}$ row and $l^{\text {th }}$ column and $f_{k, l}(x, y)$ is the DM's distinct influence function, centered at the actuator's position.

Suppose we add this DM in the pupil plane to the expression in Eq. 2.2, assuming a first order approximation for its effect and neglecting any cross terms between with DM and the aberration. Furthermore, assuming the star light has been suppressed at the high contrast region (either using a coronagraph or a shaped pupil), then the electric field in the high contrast region at the image plane is given by

$$
\widehat{E_{\mathcal{H}}}(\xi, \eta)=\widehat{A \Phi}(\xi, \eta)+i \frac{2 \pi}{\lambda} \widehat{A \phi}(\xi, \eta)
$$

where $\mathcal{H}$ denotes the high contrast region and the hat represents the Fourier Transform. Following the correction method described in [Borde' (2005)] which minimizes the total intensity of light in the high contrast region, the condition for a stationary point with respect to the heights of the actuators is given by:

$$
\sum_{m=0}^{N-1} \sum_{n=0}^{N-1} a_{m, n} \Re\left\{\left\langle\widehat{A f}_{m, n}, \widehat{A f}_{k, l}\right\rangle\right\}=\frac{\lambda}{2 \pi} \Im\left\{\left\langle\widehat{A \Phi}, \widehat{A f}_{k, l}\right\rangle\right\}
$$

for all $k, l=1,2, \ldots, N$. This condition can be put in a matrix notation and solved for the heights of the actuators. The resulting DM configuration will minimize the total intensity of light assuming a first order approximation for the DM. In order to take into account the non-linearities in the system, we may close the loop and iterate. The following section shows the simulation of this closed loop system. 


\section{Simulation}

Figure 1 shows the pupil used in these simulations. The image on the left is the shaped pupil with its ideal PSF in the center (All PSF images are given in log scale. The axes are in units of $\lambda / D)$. In order to simulate the correction method described in this paper, system aberrations were added to the model as described in Eq. 2.2. The phase aberration was simulated with $R M S=\lambda / 100$ and a $1 / f^{3 / 2}$ power law ( $f$ in this case is the spatial frequency). The amplitude aberration was simulated with $R M S=0.005$ and a $1 / f^{2}$ power law. A pinhole with a diameter of $5 * 10^{-4} \mathrm{~m}$ was added at the center of the shaped
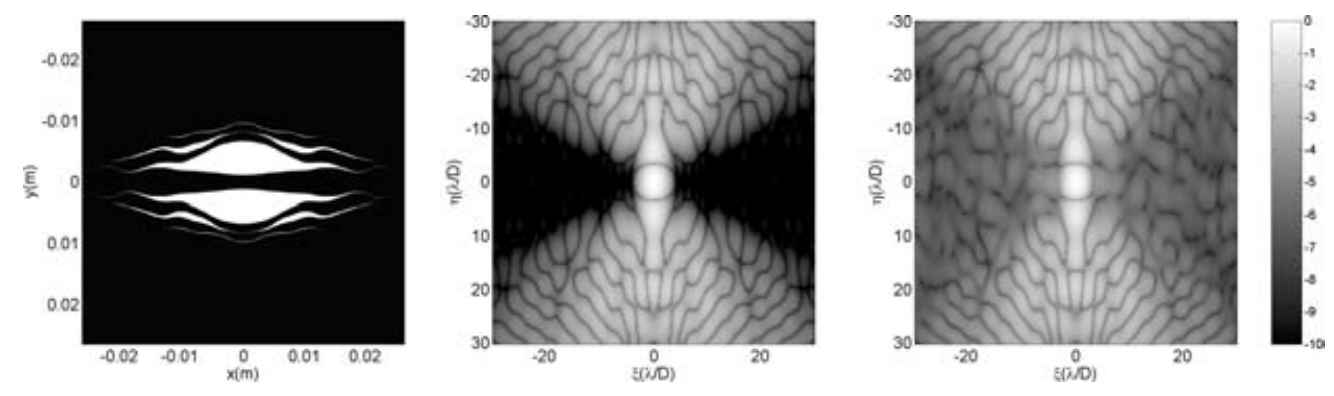

Figure 1. The shaped pupil (left) used in the simulations here and its corresponding ideal PSF (center) and the PSF of the system with aberrations (right). As seen in the figure on the right the aberrations in the system effect the contrast in the image.

pupil, as described in Eq. 2.6. The DM actuators, aligned with the pinhole were used to apply three different phase differences across the pinhole. The phase differences used in this simulation were $0, \lambda / 3$ and $2 \lambda / 3$. The following figure shows the PSF's of the system, simulating this diversity:
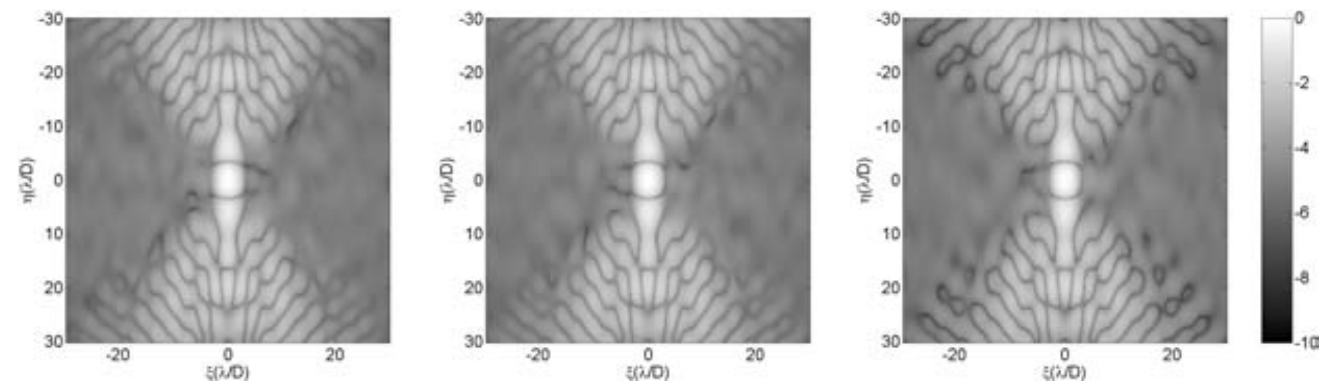

Figure 2. The three different images taken for the reconstruction part. As seen in the images, the effect of the pinhole is to add more light everywhere in the image plane. This is the reason this diversity performs well.

Simulations of this method has been successful in reconstructing the real and imaginary parts of $E_{\Phi}(\xi, \eta)$. The error in reconstruction (its absolute valued squared) is of the order of $10^{-25}$ is the dark hole region.

Figure 3 shows the PSF at the end of each iteration for the closed loop system. "Iteration 0" refers to the PSF of the system before the correction is applied. The DM was modeled with $52 \times 52$ actuators and the correction was on one side of the PSF (in order to correct for both amplitude and phase), up to $25 \lambda / D$. As seen in the figure, in this ideal case, the correction converges after the third iteration. 

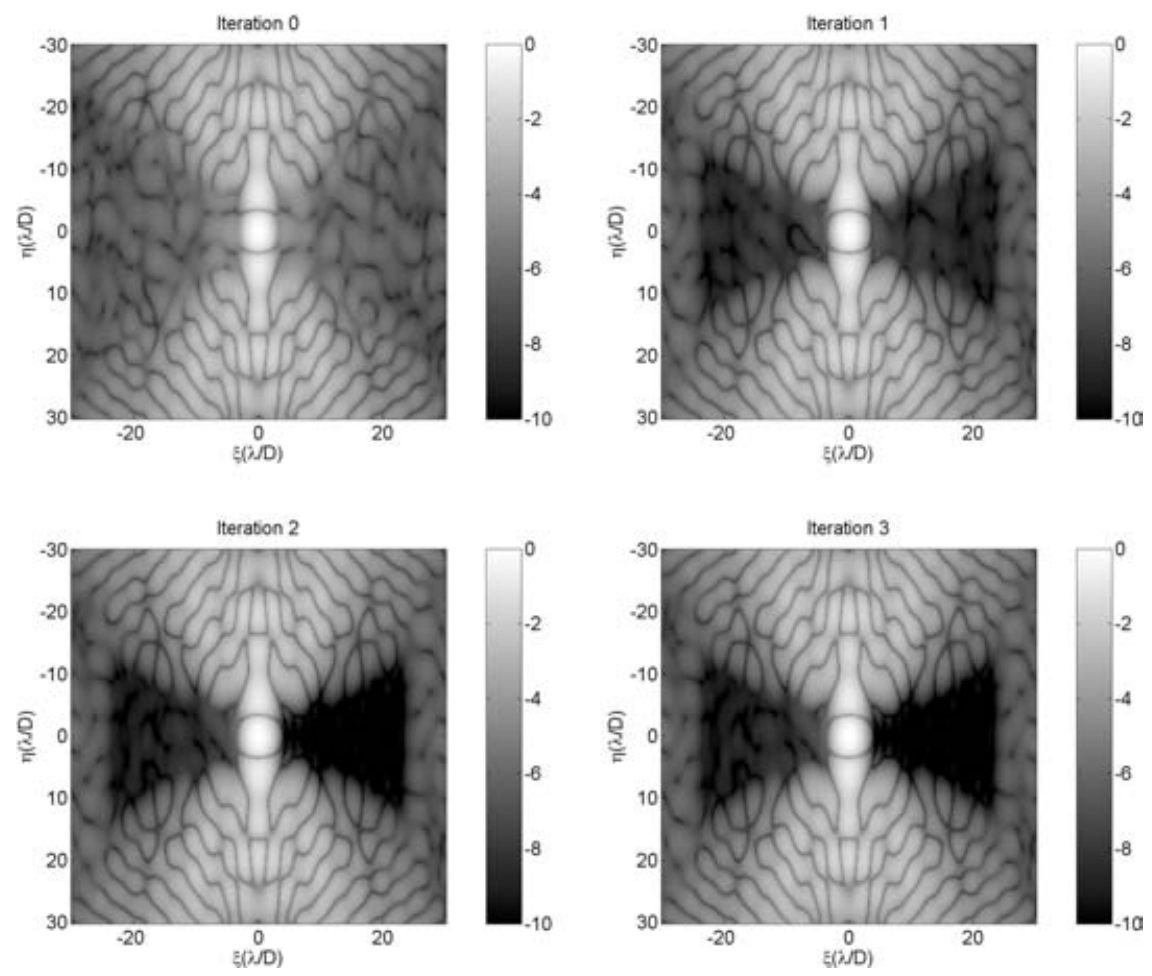

Figure 3.

\section{Algorithm Robustness}

The simulation shown above describes the ideal case. This section shows the simulation results of the closed loop method described in this paper in non-ideal cases. The first case is when the measured PSF follows a Poisson statistics for the arrival of photons at the image plane. The second case is when the shape of the DM is not exactly a sum of influence functions as assumed in the correction part (see Eq. 3.1) but an average between this model and a spline interpolation between the actuators heights. The third case is when some of the actuators fail and the DM surface at the location of the failed actuators is pinned to the substrate. Figure 4 compares the convergence of these three non-ideal cases to one another and to the ideal case.

The curve labeled "Ideal" describes the performance of the closed loop system when all the actuators are active, the DM model is exact and the measurement of the three images is deterministic. The curve labeled "Dead actuators" refers to the case when two actuators that are aligned with the open area of the shaped pupil are pinned to the substrate of the DM. The curve labeled "DM modeling error" refers to the case when the surface of the DM is a spline interpolation between the actuators' heights, rather than a sum of influence functions. The curve labeled "Photon noise" refers to the case where the PSF is the expected value of photons arriving at each CCD pixel. The measurement of the PSF is Poisson distributed. The curve labeled "All" refers to the combination case of all three modification to the model of the system. 


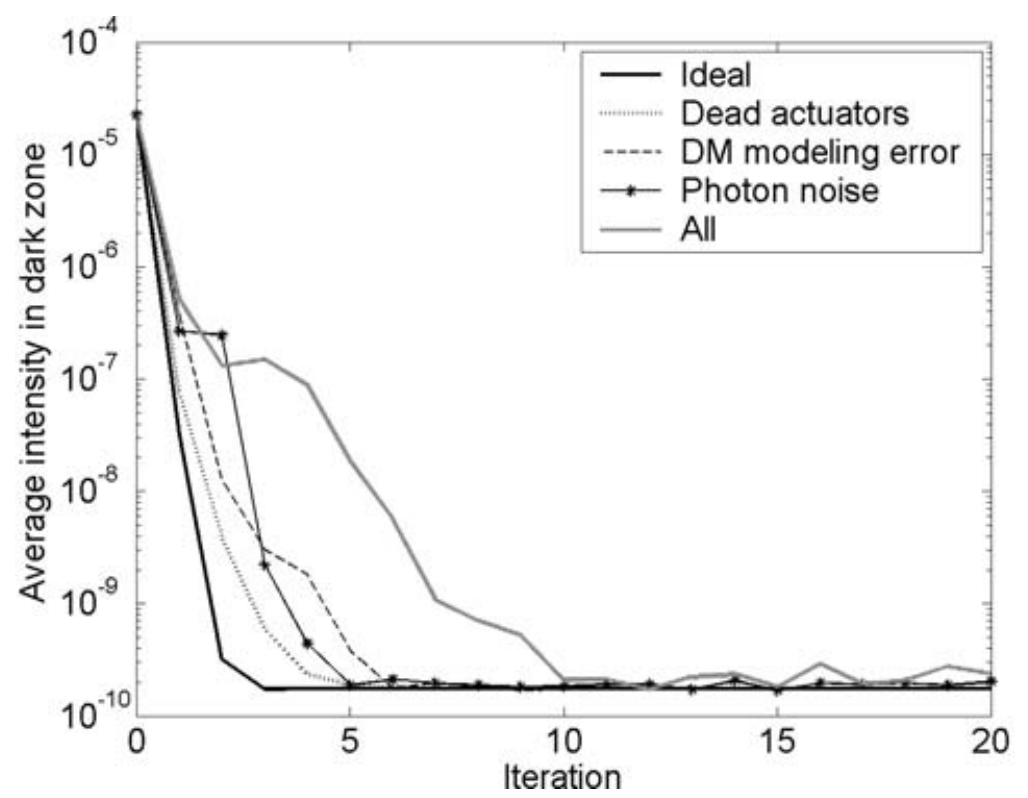

Figure 4. A plot comparing the convergence of the closed loop correction method in the presence of photon noise, deformable mirror modeling errors and defective actuators in the deformable mirror. The closed loop system was simulated in each case independently and then in the case of all modifications in effect at the same time.

As seen in the plots, the three model modifications, in the distinct cases, caused the closed loop system to converge in several more iterations. In the case of photon noise, ringing occurs in the convergence, which is expected from the stochastic nature of the system.

\section{Summary and Future Work}

This paper presents an innovative reconstruction method as part of a closed loop wavefront correction system for shaped pupil coronagraphs. This method has been shown in simulations to be effective in the presence of photon noise, deformable mirror modeling errors and overcoming defective actuators in the deformable mirror. We hope to test this reconstruction method in the lab in the near future and ultimately close the loop around it with DMs.

\section{References}

Beichman, C. A., Woolf, N. J., \& Lindensmith, C. A., "The Terrestrial Planet Finder", volume 99-3, Pasadena: JPL Publication, 1999.

Borde', P. J. \& Traub, W. A., "High-contrast imaging from space: speckle nulling in a low aberration regime", Accepted to ApJ, 2005, (astro-ph/0510597).

Give'on, A., Kasdin, N. J., Vanderbei, R. J., \& Avitzour, Y., "On representing and correcting wavefront errors in high-contrast imaging systems", Accepted to JOSA A, Oct. 2005.

Kasdin, N. J., Vanderbei, R.J., Spergel, D. N., \& Littman, M. G., "Extrasolar Planet Finding via Optimal Apodized and Shaped Pupil Coronagraphs", ApJ, 582: 1147-1161, 2003.

Poyneer, L. A. \& Macintosh, B., "Spatially filtered wave-front sensor for high-order adaptive optics", JOSA A, 21(5):810-819, May 2004.

Tyson, R. K., "Introduction to Adaptive Optics", SPIE Press, 2000. 